\title{
On the relative strengths of fragments of collection
}

\author{
Zachiri McKenzie* \\ University of Michigan-Shanghai Jiao Tong University Joint Institute, 800 Dong Chuan Road, Shanghai \\ 200240, P. R. China.
}

\begin{abstract}
Received 28 June 2018, revised 18 August 2018, accepted 23 August 2018
Published online XXXX
\end{abstract}

Let $\mathrm{M}$ be the basic set theory that consists of the axioms of extensionality, emptyset, pair, union, powerset, infinity, transitive containment, $\Delta_{0}$-separation and set foundation. This paper studies the relative strength of set theories obtained by adding fragments of the set-theoretic collection scheme to $\mathrm{M}$. We focus on two common parameterisations of the collection: $\Pi_{n}$-collection, which is the usual collection scheme restricted to $\Pi_{n}$-formulae, and strong $\Pi_{n}$-collection, which is equivalent to $\Pi_{n}$-collection plus $\Sigma_{n+1}$-separation. The main result of this paper shows that for all $n \geq 1$,

(1) $\mathrm{M}+\Pi_{n+1}$-collection $+\Sigma_{n+2}$-induction on $\omega$ proves that there exists a transitive model of Zermelo Set Theory plus $\Pi_{n}$-collection,

(2) the theory $\mathrm{M}+\Pi_{n+1}$-collection is $\Pi_{n+3}$-conservative over the theory $\mathrm{M}+$ strong $\Pi_{n}$-collection.

It is also shown that (2) holds for $n=0$ when the Axiom of Choice is included in the base theory. The final section indicates how the proofs of (1) and (2) can be modified to obtain analogues of these results for theories obtained by adding fragments of collection to a base theory (Kripke-Platek Set Theory with Infinity plus $\mathbf{V}=\mathbf{L}$ ) that does not include the powerset axiom.

Copyright line will be provided by the publisher

\section{Introduction}

In [7], Mathias systematically studies and compares a variety of subsystems of ZFC. One of the weakest systems studied in [7] is the set theory $M$ axiomatised by: extensionality, emptyset, pair, union, powerset, infinity, transitive containment, $\Delta_{0}$-separation and set foundation. This paper will expand upon some of the initial comparisons of extensions of $M$ achieved in [7] by studying the strengths of extensions of $M$ obtained by adding fragments of the set-theoretic collection scheme. The fragments of the collection scheme considered in this paper will be obtained by restricting the following alternative versions of the collection scheme to the Takahashi class $\Delta_{0}^{\wp}$ and the Lévy $\Pi_{n}$ classes:

For all formulae $\varphi(x, y, \vec{z})$ in the language of set theory,

$$
\forall \vec{z} \forall w((\forall x \in w) \exists y \varphi(x, y, \vec{z}) \Rightarrow \exists C(\forall x \in w)(\exists y \in C) \varphi(x, y, \vec{z})) .
$$

(Collection)

For all formulae $\varphi(x, y, \vec{z})$ in the language of set theory,

$$
\forall \vec{z} \forall w \exists C(\forall x \in w)(\exists y \varphi(x, y, \vec{z}) \Rightarrow(\exists y \in C) \varphi(x, y, \vec{z})) .
$$

(Strong Collection)

Both Collection and Strong Collection yield ZF when added to M. In $\S 2$, we note that, over M, the restriction of the Strong Collection scheme to $\Pi_{n}$-formulae (strong $\Pi_{n}$-collection) is equivalent to the restriction of the Collection scheme to $\Pi_{n}$-formulae ( $\Pi_{n}$-collection) plus separation for all $\Sigma_{n+1}$-formulae. This means that M plus $\Pi_{n+1}$-collection proves all instances of strong $\Pi_{n}$-collection.

One of the many achievements of [7] is showing that if $M$ is consistent, then so is $M$ plus the Axiom of Choice and strong $\Delta_{0}$-collection. In $\S 3$, we investigate the strength of adding $\Delta_{0}^{\wp}$-collection to four of the weak set theories studied in [7]. We show that if $T$ is one of the theories $\mathrm{M}, \mathrm{Mac}, \mathrm{M}+\mathrm{H}$ or MOST, then $T$ plus $\Delta_{0}^{\wp}$ collection is $\Pi_{2}^{\wp}$-conservative over $T$. As a consequence, we are able to extend the consistency results of [7] by showing that if $M$ is consistent, then so is $M$ plus the Axiom of Choice plus $\Pi_{1}$-collection.

* E-mail: zach.mckenzie@gmail.com cite this article as doi: $10.1002 /$ malq.201800044 
The results of [7] also show that the theory obtained by adding strong $\Pi_{1}$-collection to $M$ is strictly stronger than M. More specifically, $M$ plus strong $\Pi_{1}$-collection proves the consistency of Zermelo Set Theory plus $\Delta_{0}$-collection. This result and the main result of $\S 3$ are generalised in $\S 4$ to show: For all $n \geq 1$,

(1) $M$ plus $\Pi_{n+1}$-collection and the scheme of induction on $\omega$ restricted to $\Sigma_{n+2}$-formulae proves that there exists a transitive model of Zermelo Set Theory plus $\Pi_{n}$-collection,

(2) the theory $\mathrm{M}+\Pi_{n+1}$-collection is $\Pi_{n+3}$-conservative over the theory $\mathrm{M}+$ strong $\Pi_{n}$-collection.

These comparisons are achieved using techniques, developed by Pino and Ressayre in [8] (cf. also [4]), for building models of fragments of the collection scheme from chains of partially elementary submodels of the universe indexed by an ordinal, or a cut of a nonstandard ordinal, of a model of set theory.

Finally, in $\S 5$ we consider replacing the base theory M by a theory, Kripke-Platek Set Theory with the Axiom of Infinity ( $\mathrm{KPI}$ ) plus $\mathbf{V}=\mathbf{L}$, that does not include the powerset axiom. We indicate how the arguments in $\S 4$ can be adapted to obtain the following analogues of (1) and (2) above: For all $n \in \omega$,

(1) $\mathrm{KPI}+\mathbf{V}=\mathbf{L}+\Pi_{n+1}$-collection and the scheme of induction on $\omega$ restricted to $\Sigma_{n+2}$-formulae proves that there exists a transitive model of the theory $\mathrm{KPI}+\mathbf{V}=\mathbf{L}+$ strong $\Pi_{n}$-collection, and full class foundation,

(2) the theory KPI $+\mathbf{V}=\mathbf{L}+\Pi_{n+1}$-collection is $\Pi_{n+3}$-conservative over the theory KPI $+\mathbf{V}=\mathbf{L}+$ strong $\Pi_{n}$-collection.

\section{Background}

Throughout this paper $\mathcal{L}$ will denote the language of set theory. Structures will usually be denoted using uppercase calligraphy roman letters $(\mathcal{M}, \mathcal{N}, \ldots)$ and the corresponding plain font letter $(M, N, \ldots)$ will be used to denote the underlying set of that structure. If $\mathcal{M}$ is a structure, then we shall use $\mathcal{L}(\mathcal{M})$ to denote the language of $\mathcal{M}$. If $\mathcal{M}$ is an $\mathcal{L}^{\prime}$-structure where $\mathcal{L}^{\prime} \supseteq \mathcal{L}$ and $a \in M$ then we shall use $a^{*}$ to denote the class $\{x \in M \mid$ $\mathcal{M} \models(x \in a)\}$. As usual $\Delta_{0}\left(=\Sigma_{0}=\Pi_{0}\right), \Sigma_{1}, \Pi_{1}, \ldots$ will be used to denote the Lévy classes of $\mathcal{L}$-formulae, and we use $\Pi_{\infty}$ to denote the union of all of these classes (i.e., $\Pi_{\infty}=\bigcup_{n \in \omega} \Sigma_{n}=\bigcup_{n \in \omega} \Pi_{n}$ ). For all $n \in \omega$, $\Delta_{n}$ is the class of all formulae that are provably equivalent to both a $\Sigma_{n}$ formula and a $\Pi_{n}$ formula. We shall also have cause to consider the class $\Delta_{0}^{\wp}$, which is the smallest class of $\mathcal{L}$-formulae that contains all atomic formulae, contains all compound formulae formed using the connectives of first-order logic, and is closed under quantification in the form $\mathcal{Q} x \in y$ and $\mathcal{Q} x \subseteq y$ where $x$ and $y$ are distinct variables, and $\mathcal{Q}$ is $\exists$ or $\forall$. The classes $\Sigma_{1}^{\wp}, \Pi_{1}^{\wp}, \Delta_{1}^{\wp}, \ldots$ are defined inductively from the class $\Delta_{0}^{\wp}$ in the same way that the classes $\Sigma_{1}, \Pi_{1}, \Delta_{1}, \ldots$ are defined from $\Delta_{0}$. If $\Gamma$ is a class of formulae and $T$ is a theory, then we write $\Gamma^{T}$ for the class of formulae that are provably equivalent in $T$ to a formula in $\Gamma$. If $\Gamma$ is a class of formulae, then we use $\operatorname{Bool}(\Gamma)$ to denote the smallest class of formulae that contains $\Gamma$, and contains all compound formulae formed using the connectives of first-order logic. Note that for all $n \in \omega, \operatorname{Bool}\left(\Sigma_{n}\right)^{\varnothing}=\operatorname{Bool}\left(\Pi_{n}\right)^{\varnothing}$ and $\operatorname{Bool}\left(\Sigma_{n}^{\wp}\right)^{\varnothing}=\operatorname{Bool}\left(\Pi_{n}^{\wp}\right)^{\varnothing}$. If $\Gamma$ is a class of formulae, then we write $\neg \Gamma$ for the class of negations of formulae in $\Gamma$. So, for all $n \in \omega,\left(\neg \Sigma_{n}\right)^{\varnothing}=\Pi_{n}^{\varnothing}$, $\left(\neg \Pi_{n}\right)^{\varnothing}=\Sigma_{n}^{\varnothing},\left(\neg \Sigma_{n}^{\wp}\right)^{\varnothing}=\left(\Pi_{n}^{\wp}\right)^{\varnothing}$, and $\left(\neg \Pi_{n}^{\wp}\right)^{\varnothing}=\left(\Sigma_{n}^{\wp}\right)^{\varnothing}$. Let $T$ be an $\mathcal{L}^{\prime}$-theory and let $S$ be $\mathcal{L}^{\prime \prime}$-theory where $\mathcal{L}^{\prime} \subseteq \mathcal{L}^{\prime \prime}$, and let $\Gamma$ be a class of $\mathcal{L}^{\prime}$-formulae. The theory $S$ is said to be $\Gamma$-conservative over $T$ if $S$ and $T$ prove the same $\Gamma$-sentences.

Let $\mathcal{M}$ and $\mathcal{N}$ be $\mathcal{L}$-structures. If $\mathcal{M}$ is a substructure of $\mathcal{N}$ then we shall write $\mathcal{M} \subseteq \mathcal{N}$. If $\Gamma$ is a class of $\mathcal{L}$-formulae then we shall write $\mathcal{M} \prec_{\Gamma} \mathcal{N}$ if $\mathcal{M} \subseteq \mathcal{N}$ and for every $\vec{a} \in M$, $\vec{a}$ satisfies the same $\Gamma$-formulae in both $\mathcal{M}$ and $\mathcal{N}$. In the case that $\Gamma$ is $\Pi_{\infty}$ or $\Sigma_{n}$ then we shall abbreviate this notation by writing $\mathcal{M} \prec \mathcal{N}$ and $\mathcal{M} \prec_{n} \mathcal{N}$ respectively. If $\mathcal{M} \subseteq \mathcal{N}$ and for all $x \in M$ and $y \in N$,

$$
\text { if } \mathcal{N} \models(y \in x) \text { then } y \in M,
$$

then we say that $\mathcal{N}$ is an end-extension of $\mathcal{M}$ and write $\mathcal{M} \subseteq_{\mathrm{e}} \mathcal{N}$. It is well-known that if $\mathcal{M} \subseteq_{\mathrm{e}} \mathcal{N}$ then $\mathcal{M} \prec_{0} \mathcal{N}$. The following is a slight generalisation of the notion of a powerset preserving end-extension that was first studied by Forster and Kaye in [2].

Definition 2.1 Let $\mathcal{M}$ and $\mathcal{N}$ be $\mathcal{L}$-structures. We say that $\mathcal{N}$ is a powerset preserving end-extension of $\mathcal{M}$, and write $\mathcal{M} \subseteq \subseteq_{\mathrm{e}}^{\wp} \mathcal{N}$ if 
(i) $\mathcal{M} \subseteq \mathrm{e} \mathcal{N}$,

(ii) for all $x \in N$ and for all $y \in M$, if $\mathcal{N} \models(x \subseteq y)$, then $x \in M$.

Just as end-extensions preserve $\Delta_{0}$ properties, powerset preserving end-extensions preserve $\Delta_{0}^{\wp}$ properties. The following is a slight modification of a result proved in [2]:

Lemma 2.2 Let $\mathcal{M}$ and $\mathcal{N}$ be $\mathcal{L}$-structures that satisfy extensionality. If $\mathcal{M} \subseteq{ }_{\mathrm{e}}^{\wp} \mathcal{N}$, then $\mathcal{M} \prec_{\Delta_{0}^{\wp}} \mathcal{N}$.

Let $\Gamma$ be a class of $\mathcal{L}$-formulae. The following define the restriction of some commonly encountered axiom and theorem schemes of ZFC to formulae in the class $\Gamma$ :

For all $\varphi(x, \vec{z}) \in \Gamma, \forall \vec{z} \forall w \exists y \forall x(x \in y \Longleftrightarrow(x \in w) \wedge \varphi(x, \vec{z}))$.

( $\Gamma$-separation)

For all $\varphi(x, y, \vec{z}) \in \Gamma, \forall \vec{z} \forall w((\forall x \in w) \exists y \varphi(x, y, \vec{z}) \Rightarrow \exists C(\forall x \in w)(\exists y \in C) \varphi(x, y, \vec{z}))$.

( $\Gamma$-collection)

For all $\varphi(x, y, \vec{z}) \in \Gamma, \forall \vec{z} \forall w \exists C(\forall x \in w)(\exists y \varphi(x, y, \vec{z}) \Rightarrow(\exists y \in C) \varphi(x, y, \vec{z}))$.

(strong $\Gamma$-collection)

For all $\varphi(x, \vec{z}) \in \Gamma, \forall \vec{z}(\exists x \varphi(x, \vec{z}) \Rightarrow \exists y(\varphi(y, \vec{z}) \wedge(\forall x \in y) \neg \varphi(x, \vec{z}))) . \quad$ ( -foundation)

If $\Gamma=\{x \in z\}$ then we shall refer to $\Gamma$-foundation as set foundation.

For all $\varphi(x, \vec{z}) \in \Gamma, \forall \vec{z}(\varphi(\varnothing, \vec{z}) \wedge(\forall n \in \omega)(\varphi(n, \vec{z}) \Rightarrow \varphi(n+1, \vec{z})) \Rightarrow(\forall n \in \omega) \varphi(n, \vec{z}))$.

$(\Gamma$-induction on $\omega)$

We shall use $\bigcup x \subseteq x$ to abbreviate the $\Delta_{0}$-formula that says that $x$ is transitive $((\forall y \in x)(\forall z \in y)(z \in x))$. We shall also make reference to the following axioms:

$$
\begin{aligned}
& \forall u \exists T(\bigcup T \subseteq T \wedge \forall z(\bigcup z \subseteq z \wedge|z| \leq|u| \Rightarrow z \subseteq T)) . \\
& \forall x \exists y(\bigcup y \subseteq y \wedge x \subseteq y) .
\end{aligned}
$$

The following weak subsystems of ZFC are studied by Mathias in [7]:

(1) $S_{1}$ is the $\mathcal{L}$-theory with axioms: extensionality, emptyset, pair, union, set difference, and powerset.

(2) $M$ is obtained from $S_{1}$ by adding $T C o$, infinity, $\Delta_{0}$-separation, and set foundation.

(3) Mac is obtained from $M$ by adding the axiom of choice.

(4) $\mathrm{M}+\mathrm{H}$ is obtained from $\mathrm{M}$ by adding $\mathrm{H}$.

(5) $\mathrm{KPI}$ is obtained from $\mathrm{M}$ by removing powerset, and adding $\Delta_{0}$-collection and $\Pi_{1}$-foundation.

(6) $\mathrm{KP}^{\wp}$ is obtained from $\mathrm{M}$ by adding $\Delta_{0}^{\wp}$-collection and $\Pi_{1}^{\wp}$-foundation.

(7) MOST is obtained from Mac by adding $\Sigma_{1}$-separation and $\Delta_{0}$-collection.

(8) $\mathrm{Z}$ is obtained from $\mathrm{M}$ by removing $\mathrm{TC}$, and adding $\Pi_{\infty}$-separation.

(9) ZC is obtained from $Z$ by adding the axiom of choice.

In addition to these theories, we shall also use $\mathrm{MOST}^{-\mathrm{AC}}$ to refer to the theory obtained by removing the axiom of choice from MOST, and $\mathrm{M}^{-}$to refer to the theory obtained by removing the powerset axiom from M. ZF and ZFC are obtained by adding $\Pi_{\infty}$-collection (or, equivalently, strong $\Pi_{\infty}$-collection) to $M$ and Mac respectively.

We begin by collecting together some well-known relationships between fragments of induction, separation, collection, and strong collection over the weak base theory $\mathrm{M}^{-}$.

Lemma 2.3 Let $\Gamma$ be a class of $\mathcal{L}$-formulae. Let $n \in \omega$. 
(1) $\mathrm{M}^{-}+\Gamma$-foundation $\vdash \neg \Gamma$-induction on $\omega$;

(2) $\mathrm{M}^{-}+\Gamma$-separation $\vdash \operatorname{Bool}(\Gamma)$-separation;

(3) $\mathrm{M}^{-}+\Gamma$-separation $\vdash \Gamma$-foundation;

(4) $\mathrm{M}^{-}+[$strong $] \Pi_{n}^{(\wp)}$-collection $\vdash$ [strong $] \Sigma_{n+1}^{(\wp)}$-collection; and

(5) $\mathrm{M}^{-}+\Pi_{n}$-collection $\vdash \Delta_{n+1}$-separation.

Another well-known application of $\Pi_{n}$-collection is that, over $\mathrm{M}^{-}$, this scheme implies that the classes $\Sigma_{n+1}$ and $\Pi_{n+1}$ are essentially closed under bounded quantification.

Lemma 2.4 Let $\varphi(x, \vec{z})$ be a $\Sigma_{n+1}$-formula, and let $\psi(x, \vec{z})$ be a $\Pi_{n+1}$-formula. The theory $\mathrm{M}^{-}+\Pi_{n}$-collection proves that $(\forall x \in y) \varphi(x, \vec{z})$ is equivalent to a $\Sigma_{n+1}$-formula, and $(\exists x \in y) \psi(x, \vec{z})$ is equivalent to a $\Pi_{n+1}$ formula.

We also observe that for all $n \in \omega$, strong $\Pi_{n}$-collection is equivalent, over $\mathrm{M}^{-}$, to $\Pi_{n}$-collection plus $\Sigma_{n+1^{-}}$ separation. The following lemma generalises one of the equivalences reported in [7, Proposition 3.14].

Lemma 2.5 For all $n \in \omega$,

(1) $\mathrm{M}^{-}+$strong $\Pi_{n}$-collection $\vdash \Pi_{n}$-collection $+\Sigma_{n+1}$-separation

(2) $\mathrm{M}^{-}+\Pi_{n}$-collection $+\Sigma_{n+1}$-separation $\vdash$ strong $\Pi_{n}$-collection.

Proof. We first prove (1). The fact that $\mathrm{M}^{-}+$strong $\Pi_{n}$-collection proves the scheme of $\Pi_{n}$-collection is clear. We need to prove that $\mathrm{M}^{-}+$strong $\Pi_{n}$-collection proves the scheme of $\Sigma_{n+1}$-separation. It immediately follows from Lemma 2.3 that $\mathrm{M}^{-}+$strong $\Pi_{n}$-collection proves the scheme of strong $\Sigma_{n+1}$-collection and $\Pi_{n^{-}}$ separation. Work in the theory $\mathrm{M}^{-}+$strong $\Pi_{n}$-collection. Consider $\exists y \varphi(y, x, \vec{z})$ where $\varphi(y, x, \vec{z})$ is $\Pi_{n}$. Let $\vec{a}, b$ be sets. By strong $\Pi_{n}$-collection, there exists a set $C$ such that

$$
(\forall x \in b)(\exists y \varphi(y, x, \vec{a}) \Rightarrow(\exists y \in C) \varphi(y, x, \vec{a})) .
$$

Therefore, using Lemma 2.4 and $\Pi_{n}$-separation, $A=\{x \in b \mid \exists y \varphi(y, x, \vec{a})\}=\{x \in b \mid(\exists y \in C) \varphi(y, x, \vec{a})\}$ is a set. This completes the proof of (1).

We turn our attention to (2). Work in the theory $\mathrm{M}^{-}+\Pi_{n}$-collection $+\Sigma_{n+1}$-separation. Let $\varphi(x, y, \vec{z})$ be a $\Pi_{n}$-formula, and let $\vec{a}, b$ be sets. Now, $\Sigma_{n+1}$-separation implies that $A=\{x \in b \mid \exists y \varphi(x, y, \vec{a})\}$ is a set. And, $(\forall x \in A) \exists y \varphi(x, y, \vec{a})$ holds. Therefore, we can apply $\Pi_{n}$-collection to obtain a set $C$ such that $(\forall x \in A)(\exists y \in C) \varphi(x, y, \vec{a})$ holds. It now follows from the definition of $A$ that

$$
(\forall x \in b)(\exists y \varphi(x, y, \vec{a}) \text { implies }(\exists y \in C) \varphi(x, y, \vec{a})) .
$$

This completes the proof of (2).

Corollary 2.6 MOST (MOST ${ }^{-\mathrm{AC}}$ ) is the same theory as $\mathrm{Mac}+$ strong $\Delta_{0}$-collection $\left(\mathrm{M}+\right.$ strong $\Delta_{0}$-collection).

Sufficiently rich set theories such as $\mathrm{M}$ and KPI allow us to express satisfaction in set structures. The following can be found in [6] and $[1, \S$ III.1]:

Lemma 2.7 In the theory $\mathrm{KPI}$, if $\mathcal{M}$ is a set structure, $\vec{a}$ is sequence of sets, and $\varphi$ is an $\mathcal{L}(\mathcal{M})$-formula in the sense of the model whose arity agrees with the length of $\vec{a}$, then the predicate " $\mathcal{M}=\varphi[\vec{v} / \vec{a}]$ " is definable by a $\Delta_{1}$-formula.

It is noted in [7] that when powerset is present the recursions involved in the definition of satisfaction can be contained in sets even without any collection. The following is a consequence [7, Proposition 3.10]:

Lemma 2.8 In the theory $M$, if $\mathcal{M}$ is a set structure, $\vec{a}$ is sequence of sets, and $\varphi$ is an $\mathcal{L}(\mathcal{M})$-formula in the sense of the model whose arity agrees with the length of $\vec{a}$, then the predicate " $\mathcal{M} \models \varphi[\vec{v} / \vec{a}]$ " is definable and $\{\langle\ulcorner\varphi\urcorner, \vec{a}\rangle \mid \vec{a} \in M \wedge \mathcal{M} \models \varphi(\vec{a})\}$ is a set.

Equipped with these results, we can now define formulae that, in the theories $\mathrm{KPI}$ and M, express satisfaction in the universe for the Lévy classes of $\mathcal{L}$-formulae. 
Definition 2.9 Define $\operatorname{Sat}_{\Delta_{0}}(n, x)$ to be the formula

$$
\begin{aligned}
(n \in \omega) \wedge\left(n=\left\ulcorner\varphi\left(v_{1}, \ldots, v_{m}\right)\right\urcorner \text { where } \varphi \text { is } \Delta_{0}\right) \wedge\left(x=\left\langle x_{1}, \ldots, x_{m}\right\rangle\right) \wedge \\
\quad \exists N\left(\bigcup N \subseteq N \wedge\left(x_{1}, \ldots, x_{m} \in N\right) \wedge\left(\langle N, \in\rangle \models \varphi\left[x_{1}, \ldots, x_{m}\right]\right)\right) .
\end{aligned}
$$

The absoluteness of $\Delta_{0}$ properties between transitive structures and the universe, and the availability of TCo in KPI implies that the formula Sat $_{\Delta_{0}}$ is equivalent, in the theory KPI, to the formula

$$
\begin{aligned}
& (n \in \omega) \wedge\left(n=\left\ulcorner\varphi\left(v_{1}, \ldots, v_{m}\right)\right\urcorner \text { where } \varphi \text { is } \Delta_{0}\right) \wedge\left(x=\left\langle x_{1}, \ldots, x_{m}\right\rangle\right) \wedge \\
& \quad \forall N\left(\bigcup N \subseteq N \wedge\left(x_{1}, \ldots, x_{m} \in N\right) \Rightarrow\left(\langle N, \in\rangle \models \varphi\left[x_{1}, \ldots, x_{m}\right]\right)\right) .
\end{aligned}
$$

Therefore, Lemma 2.7 implies that $\operatorname{Sat}_{\Delta_{0}}(n, x)$ is $\Delta_{1}^{\mathrm{KPI}}$, and $\operatorname{Sat}_{\Delta_{0}}(n, x)$ expresses satisfaction for $\Delta_{0}$-formulae in the theories KPI and M. We can now inductively define formulae $\operatorname{Sat}_{\Sigma_{m}}(n, x)$ and $\operatorname{Sat}_{\Pi_{m}}(n, x)$ that express satisfaction for formulae in the classes $\Sigma_{m}$ and $\Pi_{m}$.

Definition 2.10 The formulae $\operatorname{Sat}_{\Sigma_{m}}(n, x)$ and $\operatorname{Sat}_{\Pi_{m}}(n, x)$ are defined inductively. Define $\operatorname{Sat}_{\Sigma_{m+1}}(n, x)$ to be the formula

$$
\begin{aligned}
\exists \vec{y} \exists k \exists b\left(\left(n=\left\ulcorner\exists \vec{u} \varphi\left(\vec{u}, v_{1}, \ldots, v_{\ell}\right)\right\urcorner \text { where } \varphi \text { is } \Pi_{m}\right) \wedge\left(x=\left\langle x_{1}, \ldots, x_{\ell}\right\rangle\right)\right. \\
\left.\wedge\left(b=\left\langle\vec{y}, x_{1}, \ldots, x_{\ell}\right\rangle\right) \wedge\left(k=\left\ulcorner\varphi\left(\vec{u}, v_{1}, \ldots, v_{\ell}\right)\right\urcorner\right) \wedge \operatorname{Sat}_{\Pi_{m}}(k, b)\right) .
\end{aligned}
$$

Define $\operatorname{Sat}_{\Pi_{m+1}}(n, x)$ to be the formula

$$
\begin{aligned}
& \forall \vec{y} \forall k \forall b\left(\left(n=\left\ulcorner\forall \vec{u} \varphi\left(\vec{u}, v_{1}, \ldots, v_{\ell}\right)\right\urcorner \text { where } \varphi \text { is } \Sigma_{m}\right)\right. \wedge\left(x=\left\langle x_{1}, \ldots, x_{\ell}\right\rangle\right) \\
&\left.\wedge\left(\left(b=\left\langle\vec{y}, x_{1}, \ldots, x_{\ell}\right\rangle\right) \wedge\left(k=\left\ulcorner\varphi\left(\vec{u}, v_{1}, \ldots, v_{\ell}\right)\right\urcorner\right) \Rightarrow \operatorname{Sat}_{\Sigma_{m}}(k, b)\right)\right) .
\end{aligned}
$$

The formula $\operatorname{Sat}_{\Sigma_{m}}(n, x)$ (respectively $\left.\operatorname{Sat}_{\Pi_{m}}(n, x)\right)$ is $\Sigma_{m}^{\mathrm{KPI}}\left(\Pi_{m}^{\mathrm{KPI}}\right.$, respectively), and, in the theories $\mathrm{KPI}$ and $\mathrm{M}$, expresses satisfaction for $\Sigma_{m}$-formulae $\left(\Pi_{m}\right.$-formulae, respectively).

Another important feature of the theory $\mathrm{KPI}$ is its ability to construct $\mathbf{L}$. The following can be found in [6] and [1, Chapter II]:

Theorem 2.11 (KPI) The function $\alpha \mapsto \mathbf{L}_{\alpha}$, where $\alpha$ is an ordinal, is total and $\Delta_{1}$.

As is usual, we use $\mathbf{V}=\mathbf{L}$ to abbreviate the expression that says that every set is the member of some $\mathbf{L}_{\alpha}$ $\left(\forall x \exists \alpha\left(\left(\alpha\right.\right.\right.$ is an ordinal $\left.\left.\left.\wedge\left(x \in \mathbf{L}_{\alpha}\right)\right)\right)\right)$.

We now turn to noting some of the properties of the theories $\mathrm{M}$, Mac, M $+\mathrm{H}$ and MOST that are established in [7]. The following useful fact is a consequence of [1, Theorem I.6.1.]:

Lemma 2.12 The theory KPI proves TCo.

We also record the following consequence of [7, Theorem Scheme 6.9(i)]:

Theorem 2.13 The theory $\mathrm{M}$ proves all instances of $\Delta_{0}^{\wp}$-separation.

$[7, \S 2]$ shows that by considering classes of well-founded extensional relations in a model of $\mathrm{M}$ one can obtain a model of $\mathrm{M}+\mathrm{H}$.

Theorem 2.14 (Mathias) If $\mathrm{M}$ is consistent, then so is $\mathrm{M}+\mathrm{H}$.

$[7, \S 3]$ establishes a variety of consequences of $\mathrm{H}$ over the theories $\mathrm{M}$ and Mac. A key observation of this section is that the theory MOST is exactly Mac $+\mathrm{H}$.

Lemma 2.15 MOST is the same theory as $\mathrm{Mac}+\mathrm{H}$.

The following useful consequences of the theory MOST (= Mac + strong $\Delta_{0}$-collection) are also proved in $[7, \S 3]$ :

Lemma 2.16 The theory MOST proves 
(i) every well-ordering is isomorphic to an ordinal,

(ii) every well-founded extensional relation is isomorphic to a transitive set,

(iii) for all cardinals $\kappa, \kappa^{+}$exists,

(iv) for all cardinals $\kappa, \mathbf{H}_{\kappa}$ exists.

$[7, \S 4]$ establishes that the theory $M+\mathrm{H}$ is capable of building Gödel's L. Combined with Theorems 2.14 \& 2.15 this yields that following consistency result:

Theorem 2.17 (Mathias; [7, Theorem 1]) If $\mathrm{M}$ is consistent, then so is MOST $+\mathbf{V}=\mathbf{L}$.

The classes $\Delta_{0}^{\wp}, \Sigma_{1}^{\wp}, \Pi_{1}^{\wp}, \ldots$ are introduced and studied by Takahashi in [10] where it is shown that for all $n \geq 1,\left(\Sigma_{n}^{\wp}\right)^{\mathrm{ZFC}}=\Sigma_{n+1}^{\mathrm{ZFC}},\left(\Pi_{n}^{\wp}\right)^{\mathrm{ZFC}}=\Pi_{n+1}^{\mathrm{ZFC}}$, and $\left(\Delta_{n}^{\wp}\right)^{\mathrm{ZFC}}=\Delta_{n+1}^{\mathrm{ZFC}}$. The following calibration of Takahashi's result appears as [7, Proposition Scheme 6.12]:

Lemma 2.18 (Takahashi) $\Sigma_{1} \subseteq\left(\Delta_{1}^{\wp}\right)^{\text {MOST }}$ and $\Delta_{0}^{\wp} \subseteq \Delta_{2}^{S_{1}}$.

This yields the following refined version of [10, Theorem 6]:

Theorem 2.19 (Takahashi) For all $n \geq 1, \Sigma_{n+1} \subseteq\left(\Sigma_{n}^{\wp}\right)^{\mathrm{MOST}}, \Pi_{n+1} \subseteq\left(\Pi_{n}^{\wp}\right)^{\mathrm{MOST}}, \Delta_{n+1} \subseteq\left(\Delta_{n}^{\wp}\right)^{\mathrm{MOST}}$, $\Sigma_{n}^{\wp} \subseteq \Sigma_{n+1}^{\mathrm{S}_{1}}, \Pi_{n}^{\wp} \subseteq \Pi_{n+1}^{\mathrm{S}_{1}}$, and $\Delta_{n}^{\wp} \subseteq \Delta_{n+1}^{\mathrm{S}_{1}}$.

Lemmas $2.3 \& 2.5$ and Theorem 2.19 now show:

Corollary 2.20 The theory $\mathrm{M}+$ strong $\Pi_{1}$-collection proves every axiom of $\mathrm{KP}^{\wp}$.

In [7], Mathias proves a $\Sigma_{1}^{\wp}$-Recursion Theorem in the theory KP ${ }^{\wp}$. The following appear as [7, Lemma 6.25 \& Theorem 6.26]:

Lemma 2.21 If $F$ is a total $\Sigma_{1}^{\wp}$-definable class function, then the formula $y=F(x)$ is $\Delta_{1}^{\wp}$.

Theorem 2.22 $\left(\mathrm{KP}^{\wp}\right)$ Let $G$ be a $\Sigma_{1}^{\wp}$-definable class. If $G$ is a total function, then there exists a $\Sigma_{1}^{\wp}$-definable total class function $F$ such that for all $x, F(x)=G(F\lceil x)$.

The fact that we have access to Theorem 2.22 in the theory $\mathrm{M}+$ strong $\Pi_{1}$-collection yields:

Corollary 2.23 The theory $\mathrm{M}+$ strong $\Pi_{1}$-collection proves that for all ordinals $\alpha, \mathbf{V}_{\alpha}$ is a set. Moreover, the formula " $x=\mathbf{V}_{\alpha}$ " with free variables $x$ and $\alpha$ is equivalent to a $\Delta_{1}^{\wp}$-formula.

Results proved in [7] also reveal that the theory $M+$ strong $\Pi_{1}$-collection is capable of proving the consistency of Zermelo Set Theory plus $\Delta_{0}$-collection. Mathias [7, Lemma 6.31] shows that the theory obtained by strengthening KP with an axiom that asserts the existence of $\mathbf{V}_{\alpha}$ for every ordinal $\alpha$ is capable of proving the consistency of $Z$. The fact that $\mathrm{KP}^{\wp}$ is equipped with enough recursion to prove the existence of $\mathbf{V}_{\alpha}$ for every $\alpha$ [7, Proposition 6.28] thus yields:

Theorem 2.24 (Mathias) The theory $\mathrm{KP}^{\wp}$ proves that there exists a transitive model of $\mathrm{Z}$.

Mathias [7, Theorem 5] also shows that all of the axioms of KP plus $\mathbf{V}=\mathbf{L}$ can be consistently added to Z. In particular:

Theorem 2.25 (Mathias) If $\mathrm{Z}$ is consistent, then so is $\mathrm{Z}+\Delta_{0}$-collection $+\mathbf{V}=\mathbf{L}$.

Theorems $2.24 \& 2.25$ now yield:

Corollary 2.26 KP $\vdash \operatorname{Con}\left(Z+\Delta_{0}\right.$-collection $\left.+\mathbf{V}=\mathbf{L}\right)$.

\section{The strength of $\Delta_{0}^{\wp}$-collection}

In this section we investigate the strength of adding $\Delta_{0}^{\wp}$-collection to subsystems of set theory studied in [7]. We show that if $T$ is one of the theories $\mathrm{M}, \mathrm{M}+\mathrm{H}$, Mac or MOST, then the theory obtained by adding $\Delta_{0}^{\wp}$-collection to $T$ is $\Pi_{2}^{\wp}$-conservative over $T$. Combined with Theorems $2.17 \& 2.19$, this shows that if $\mathrm{M}$ is consistent, then so is MOST $+\Pi_{1}$-collection. If $u$ is a set, then we shall use $\mathbf{H}_{\leq|u|}$ to denote the set $\{x|| \operatorname{TC}(\{x\})|\leq| u \mid\}$.

Lemma 3.1 The theory $\mathrm{M}+\mathrm{H}$ proves that for all sets $u, \mathbf{H}_{\leq|u|}$ exists. 
Proof. Work in the theory $\mathrm{M}+\mathrm{H}$. Let $u$ be a set. Using $\mathrm{H}$, let $T$ be a set such that

$$
\forall z(\bigcup z \subseteq z \wedge|z| \leq|u| \Rightarrow z \subseteq T) .
$$

Note that if $x$ is a set such that $|\mathrm{TC}(\{x\})| \leq|u|$, then $\mathrm{TC}(\{x\}) \subseteq T$ and so $x \in T$. Moreover, if $|\mathrm{TC}(\{x\})| \leq$ $|u|$, then $\operatorname{TC}(\{x\}) \in \wp(T)$ and the injection witnessing $|\mathrm{TC}(\{x\})| \leq|u|$ is in $\wp(T \times u)$. Therefore $\Delta_{0}$-separation implies that $\mathbf{H}_{\leq|u|}$ exists.

The following is immediate from the definition of $\mathbf{H}_{\leq|u|}$ :

Lemma 3.2 The theory $\mathrm{M}+\mathrm{H}$ proves that if $u, x, y$ are sets, then

(i) if $x \in y \in \mathbf{H}_{\leq|u|}$, then $x \in \mathbf{H}_{\leq|u|}$, and

(ii) if $x \subseteq y \in \mathbf{H}_{\leq|u|}$, then $x \in \mathbf{H}_{\leq|u|}$.

Definition 3.3 Let $n \in \omega$ and let $u$ be a set. We say that $f$ is an $n$-good $|u|-H$-approximation if

(i) $f$ is a function and $\operatorname{dom}(f)=n+1$,

(ii) $f(\varnothing)=\mathbf{H}_{\leq|u|}$,

(iii) $(\forall k \in n+1) \exists v\left(f(k)=\mathbf{H}_{\leq|v|}\right)$, and

(iv) $(\forall k \in n)(f(k) \in f(k+1))$.

We first observe that in any model of $\mathrm{M}+\mathrm{H}$ there exists an $n$-good $|u|-H$-approximation for every externally finite $n$ and every set $u$ in the model.

Lemma 3.4 Let $n \in \omega$. If $\mathcal{M} \models \mathrm{M}+\mathrm{H}$ and $u \in M$, then $\mathcal{M}=\exists f(f$ is an $n$-good $|u|-H$-approximation $)$.

Proof. Let $\mathcal{M}=\langle M, \in \mathcal{M}\rangle$ be such that $\mathcal{M} \models \mathrm{M}+\mathrm{H}$ and let $u \in M$. We prove, by external induction on $\omega$, that for all $n \in \omega, \mathcal{M} \models \exists f(f$ is an $n$-good $|u|-H$-approximation). It follows from Lemma 3.1 that $\mathcal{M} \models \exists f(f$ is a 0 -good $|u|-H$-approximation). Suppose that the lemma is false, and $k \in \omega$ is least such that $\mathcal{M} \models \neg \exists f(f$ is a $(k+1)$-good $|u|-H$-approximation). Work inside $\mathcal{M}$. Let $f$ be a $k$-good $|u|-H$-approximation. Let $v=f(k) \cup\{f(k)\}$. It follows from Definition 3.3(iii) \& Lemma 3.2 that $v=\mathrm{TC}(\{f(k)\})$. Therefore $g=f \cup\left\{\left\langle k+1, \mathbf{H}_{\leq|v|}\right\rangle\right\}$ is a $(k+1)$-good $|u|-H$-approximation, which is a contradiction.

In the proof of the following result we obtain models of $\Delta_{0}^{\wp}$-collection by considering a cut of an $n$-good $|u|$ $H$-approximation of nonstandard length. This idea of obtaining "more" collection from a cut of a nonstandard model of set theory also appears in Ressayre's work on limitations of extensions of Kripke-Platek Set Theory [8] (cf. also [4]) and Friedman's work [3] on the standard part of countable non-standard models of set theory.

Theorem 3.5 (i) The theory $\mathrm{M}+\mathrm{H}+\Delta_{0}^{\wp}$-collection is $\Pi_{2}^{\wp}$-conservative over the theory $\mathrm{M}+\mathrm{H}$.

(ii) The theory MOST $+\Pi_{1}$-collection is $\Pi_{3}$-conservative over the theory MOST.

Proof. To prove (i) it is sufficient to show that every $\Sigma_{2}^{\wp}$-sentence that is consistent with $\mathrm{M}+\mathrm{H}$ is also consistent with $\mathrm{M}+\mathrm{H}+\Delta_{0}^{\wp}$-collection. Suppose that $\exists \vec{x} \forall \vec{y} \vartheta(\vec{x}, \vec{y})$, where $\vartheta(\vec{x}, \vec{y})$ is a $\Delta_{0}^{\wp}$-formulae, is consistent with $\mathrm{M}+\mathrm{H}$. Let $\mathcal{M}=\left\langle M, \in^{\mathcal{M}}\right\rangle$ be a recursively saturated model of $\mathrm{M}+\mathrm{H}+\exists \vec{x} \forall \vec{y} \vartheta(\vec{x}, \vec{y})$. Let $\vec{a} \in M$ be such $\mathcal{M} \models \forall \vec{y} \vartheta(\vec{a}, \vec{y})$ and let $u \in M$ be such that $\vec{a} \in u$. Consider the type

$$
\Xi(x, u)=\{x \in \omega\} \cup\{x>n \mid n \in \omega\} \cup\{\exists f(f \text { is an } x \text {-good } u \text { - } H \text {-approximation })\} .
$$

By Lemma 3.4, $\Xi(x, u)$ is finitely realised in any model of $\mathrm{M}+\mathrm{H}$, and so there exists $k \in M$ such that $\Xi(k, u)$ is satisfied in $\mathcal{M}$. Note that $k$ is a nonstandard element of $\omega^{\mathcal{M}}$. Let $f \in M$ be such that

$$
\mathcal{M}=(f \text { is a } k \text {-good } u \text { - } H \text {-approximation }) .
$$

Define $\mathcal{N}=\left\langle N, \in^{\mathcal{N}}\right\rangle$ by $N=\bigcup_{n \in \omega} f\left(n^{\mathcal{M}}\right)^{*}$ and $\in^{\mathcal{N}}$ is the restriction of $\in^{\mathcal{M}}$ to $N$. We claim that $\mathcal{N}$ satisfies $\mathrm{M}+\mathrm{H}+\Delta_{0}^{\wp}$-collection $+\exists \vec{x} \forall \vec{y} \vartheta(\vec{x}, \vec{y})$. Note that $\mathcal{N} \subseteq_{\mathrm{e}}^{\wp} \mathcal{M}$ and $\vec{a} \in N$, so $\mathcal{N} \models \exists \vec{x} \forall \vec{y} \vartheta(\vec{x}, \vec{y})$. Let $x \in N$. Let 
$n \in \omega$ be such that $\mathcal{M} \models\left(x \in f\left(n^{\mathcal{M}}\right)\right)$. Therefore $\mathcal{M} \models\left(\wp(x) \subseteq f\left(n^{\mathcal{M}}\right)\right)$ and $f\left(n^{\mathcal{M}}\right) \in\left(f\left((n+1)^{\mathcal{M}}\right)\right)^{*} \subseteq$ $N$. It now follows from Definition 3.3 that $\wp^{\mathcal{M}}(x) \in N$. Therefore $\mathcal{N} \models$ (powerset) and for all $x \in N$, $\wp^{\mathcal{N}}(x)=\wp^{\mathcal{M}}(x)$. It is now clear that $\mathcal{N} \models \mathrm{M}$.

We turn to showing that $\mathrm{H}$ holds in $\mathcal{N}$. Let $u \in N$. Let $n \in \omega$ be such that $u \in f\left(n^{\mathcal{M}}\right)^{*}$. By Definition 3.3, there exists $v \in M$ such that $\mathcal{M} \models\left(f\left(n^{\mathcal{M}}\right)=\mathbf{H}_{\leq|v|}\right)$, and so $\mathcal{M} \models(|u| \leq|v|)$. Now, working inside $\mathcal{N}$, if $z$ is transitive with $|z| \leq|u|$, then $|z| \leq|v|$ and so $z \in f\left(n^{\mathcal{M}}\right)$. Therefore

$$
\mathcal{N} \models \forall z\left(\bigcup z \subseteq z \wedge|z| \leq|u| \Rightarrow z \in f\left(n^{\mathcal{M}}\right)\right)
$$

and so $\mathrm{H}$ holds in $\mathcal{N}$.

We are left to show that $\mathcal{N}$ satisfies $\Delta_{0}^{\wp}$-collection. We make use of the following property of $\mathcal{N}$ :

Claim 3.6 If $C \in M$ and $C^{*} \subseteq N$, then $C \in N$.

Proof of Claim 3.6. Suppose, for a contradiction, that $C \in M, C^{*} \subseteq N$ and $C \notin N$. Note that if $n \in k^{*}$ is nonstandard, then $C^{*} \subseteq f(n)^{*}$ and $\mathcal{M} \models(C \in f(n+1))$. Therefore, working inside $\mathcal{M}$, the set $A=\{n \in k \mid C \notin f(n)\}$ defines the standard $\omega$, which is a contradiction.

Now, let $\varphi(x, y, \vec{z})$ be a $\Delta_{0}^{\wp}$-formula. Let $\vec{d}, b \in N$ be such that $\mathcal{N} \models(\forall x \in b) \exists y \varphi(x, y, \vec{d})$. The following formula is a $\Delta_{0}^{\wp}$-formula with parameters $\vec{d}, k$ and $f$ :

$$
\varphi(x, y, \vec{d}) \wedge(\forall n \in k)(y \notin f(n) \Rightarrow \neg(\exists w \in f(n)) \varphi(x, w, \vec{d})) .
$$

So, by $\Delta_{0}^{\wp}$-absoluteness,

$$
\mathcal{M}=(\forall x \in b)(\exists y \in f(k))(\varphi(x, y, \vec{d}) \wedge(\forall n \in k)(y \notin f(n) \Rightarrow \neg(\exists w \in f(n)) \varphi(x, w, \vec{d}))) .
$$

Working inside $\mathcal{M}, \Delta_{0}^{\wp}$-separation (Theorem 2.13) implies that

$$
C=\{\langle x, y\rangle \in b \times f(k) \mid \varphi(x, y, \vec{d}) \wedge(\forall n \in k)(y \notin f(n) \Rightarrow \neg(\exists w \in f(n)) \varphi(x, w, \vec{d}))
$$

is a set. And $\Delta_{0}^{\wp}$-absoluteness implies that $C^{*} \subseteq N$. Therefore $C \in N$. Working inside $\mathcal{N}$, let $B=\operatorname{rng}(C)$. So,

$$
\mathcal{N} \models(\forall x \in b)(\exists y \in B) \varphi(x, y, \vec{d}),
$$

which shows that $\mathcal{N} \models \Delta_{0}^{\wp}$-collection.

To see that (ii) holds observe that if the Axiom of Choice holds in $\mathcal{M}$ in the proof of (i), then it also holds in $\mathcal{N}$. It then follows from Theorem 2.19 that $\mathcal{N}$ also satisfies $\Pi_{1}$-collection, and we get $\Pi_{3}$-conservativity.

Theorem 3.5 combined with Theorems 2.17 shows that the consistency M implies the consistency of MOST + $\Pi_{1}$-collection.

Corollary 3.7 If $\mathrm{M}$ is consistent, then so is $\mathrm{MOST}+\Pi_{1}$-collection (= Mac $+\Pi_{1}$-collection).

The argument used in the proof of Theorem 3.5 can also be used to show that that the theories $\mathrm{M}+\Delta_{0}^{\wp}$-collection and $\mathrm{Mac}+\Delta_{0}^{\wp}$-collection are $\Pi_{2}^{\wp}$-conservative over the theories $\mathrm{M}$ and $\mathrm{Mac}$, respectively. To see this we introduce a modification of Definition 3.3:

Definition 3.8 Let $n \in \omega$ and let $u$ be a set. We say that $f$ is an $n$-good $u$ - $\wp$-approximation if

(i) $f$ is a function and $\operatorname{dom}(f)=n+1$,

(ii) $f(\varnothing)=\mathrm{TC}(u)$, and

(iii) $(\forall k \in n)(f(k+1)=\wp(f(k)))$.

An $n$-good $u$ - $\wp$-approximation is a sequence $\wp(v), \wp(\wp(v)), \ldots$ where $v$ is the transitive closure of $u$. The same argument that was used to prove Lemma 3.4 shows that in any model of $M$, any such sequence with externally finite length is guaranteed to exist. 
Lemma 3.9 Let $n \in \omega$. If $\mathcal{M} \models M$ and $u \in M$, then $\mathcal{M} \models \exists f(f$ is an n-good u-ø-approximation $)$.

Replacing the $n$-good $|u|-H$-approximations in the proof of Theorem 3.5 now shows that adding $\Delta_{0}^{\wp}$-collection to $\mathrm{M}$ or Mac does not prove any new $\Pi_{2}^{\wp}$-sentences.

Theorem 3.10 (i) The theory $\mathrm{M}+\Delta_{0}^{\wp}$-collection is $\Pi_{2}^{\wp}$-conservative over the theory $\mathrm{M}$.

(ii) The theory $\mathrm{Mac}+\Delta_{0}^{\wp}$-collection is $\Pi_{2}^{\wp}$-conservative over the theory Mac.

Theorems $3.5 \& 3.10$ highlight a mistake in the final sentence of [7, Metatheorem 9.41] and the final clause, starting after the colon, of [7, Theorem 16] (which paraphrases [7, Metatheorem 9.41]). This erroneous assertion is used by the author in [5] to claim that the theory Mac $+\Delta_{0}^{\wp}$-collection represents a new lower-bound on the consistency strength of the theory NFU + AxCount $\leq$. Theorem 3.5 now shows that Mac $+\Delta_{0}^{\wp}$-collection does not represent an improvement on previously known lower-bounds on the consistency strength of NFU $+\mathrm{AxCount}_{\leq}$.

\section{The strength of $\Pi_{n}$-collection over $\mathrm{M}$}

In this section we generalise and expand upon Theorem 3.5 to show for all $n \geq 1$,

(1) the theory $\mathrm{M}+\Pi_{n+1}$-collection is $\Pi_{n+3}$-conservative over the theory $\mathrm{M}+$ strong $\Pi_{n}$-collection,

(2) the theory $\mathrm{M}+\Pi_{n+1}$-collection $+\Sigma_{n+2}$-induction on $\omega$ proves that there exists a transitive model of $\mathrm{Z}+$ $\Pi_{n}$-collection.

The main tool used in the proof of these results will be the following modification and generalisation of Definition 3.3:

Definition 4.1 Let $n, m \in \omega$, and let $\alpha$ be an ordinal. We say that $f$ is an $n$-good $\langle m+1, \alpha\rangle$-submodel approximation if

(i) $f$ is a function and $\operatorname{dom}(f)=n+1$,

(ii) $f(\varnothing)=\mathbf{V}_{\alpha}$,

(iii) $(\forall k \in n+1) \exists \beta\left((\beta\right.$ is an ordinal $\left.) \wedge f(k)=\mathbf{V}_{\beta}\right)$,

(iv) $(\forall k \in n)(\forall \ell \in \omega)(\forall a \in f(k+1))\left(\left(\langle f(k+1), \in\rangle \models \operatorname{Sat}_{\Pi_{m}}(\ell, a)\right) \Rightarrow \operatorname{Sat}_{\Pi_{m}}(\ell, a)\right)$, and

(v) $(\forall k \in n)(\forall \ell \in \omega)(\forall a \in f(k))\left(\operatorname{Sat}_{\Sigma_{m+1}}(\ell, a) \Rightarrow\left(\langle f(k+1), \in\rangle \models \operatorname{Sat}_{\Sigma_{m+1}}(\ell, a)\right)\right)$.

An $n$-good $\langle m+1, \alpha\rangle$-submodel approximation is a sequence $\left\langle\mathbf{V}_{\beta_{0}}, \ldots, \mathbf{V}_{\beta_{n}}\right\rangle$ such that $\mathbf{V}_{\beta_{0}}=\mathbf{V}_{\alpha}$ (condition (ii)), for all $0 \leq \ell<k, \beta_{\ell} \leq \beta_{k}$ (condition (v) applied to the $\Sigma_{1}$-formula " $\exists v(a \in v)$ )"), each $\mathbf{V}_{\beta_{k}}(1 \leq k \leq n)$ is a $\Pi_{m}$-elementary submodel of the universe (condition (iv)), each $\mathbf{V}_{\beta_{k+1}}$ satisfies the same $\Sigma_{m+1}$-formulae with parameters from $\mathbf{V}_{\beta_{k}}$ as the universe (condition (v)). Note that if an infinite sequence $\left\langle\mathbf{V}_{\beta_{0}}, \mathbf{V}_{\beta_{1}}, \ldots\right\rangle$ is such that for every $n \in \omega$, the first $n+1$ elements of this sequence form an $n$-good $\langle m+1, \alpha\rangle$-submodel approximation, then $\bigcup_{n \in \omega} \mathbf{V}_{\beta_{n}}$ is a $\Pi_{m+1}$-elementary submodel of the universe.

We make the following observations about the complexity of Definition 4.1:

(1) The formula " $f$ is a function and $\operatorname{dom}(f)=n+1$ " is $\Delta_{0}$ with parameters $f$ and $n$.

(2) The formula " $f(\varnothing)=\mathbf{V}_{\alpha}$ " is $\Delta_{0}$ with parameters $f$ and $\mathbf{V}_{\alpha}$.

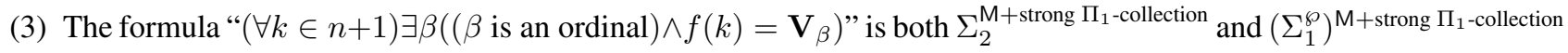
with parameters $f$ and $n$.

(4) For all $m \in \omega$, the formula

$$
(\forall k \in n)(\forall \ell \in \omega)(\forall a \in f(k+1))\left(\left(\langle f(k+1), \in\rangle \models \operatorname{Sat}_{\Pi_{m}}(\ell, a)\right) \Rightarrow \operatorname{Sat}_{\Pi_{m}}(\ell, a)\right)
$$

is $\Pi_{\max (1, m)}^{\mathrm{KPI}}$ with parameters $f$ and $n$.

Copyright line will be provided by the publisher

This article is protected by copyright. All rights reserved. 
(5) For all $m \in \omega$, the formula

$$
(\forall k \in n)(\forall \ell \in \omega)(\forall a \in f(k))\left(\operatorname{Sat}_{\Sigma_{m+1}}(\ell, a) \Rightarrow\left(\langle f(k+1), \in\rangle \models \operatorname{Sat}_{\Sigma_{m+1}}(\ell, a)\right)\right)
$$

is $\Pi_{m+1}^{\mathrm{KPI}}$ with parameters $f$ and $n$.

In light of these observations we introduce specific notion for the formulae that say that $f$ is an $n$-good $\langle m+1, \alpha\rangle$ submodel approximation.

Definition 4.2 Let $\alpha$ be an ordinal and let $m \in \omega$. We write $\Psi_{m}\left(n, f, \mathbf{V}_{\alpha}\right)$ for the formula, with free variables $f$ and $n$, and parameter $\mathbf{V}_{\alpha}$, that the theory $\mathbf{M}+$ strong $\Pi_{1}$-collection proves asserts that $f$ in an $n$-good $\langle m+1, \alpha\rangle$-submodel approximation, and such that $\Psi_{0}\left(n, f, \mathbf{V}_{\alpha}\right)$ is $\Sigma_{2}, \Psi_{1}\left(n, f, \mathbf{V}_{\alpha}\right)$ is $\operatorname{Bool}\left(\Sigma_{2}\right)$, and if $m>1$, $\Psi_{m}\left(n, f, \mathbf{V}_{\alpha}\right)$ is $\Pi_{m+1}$.

Lemma 4.3 The theory $M+$ strong $\Pi_{1}$-collection proves that for all ordinals $\alpha$ and for all $n \in \omega$, there exists an $n$-good $\langle 1, \alpha\rangle$-submodel approximation.

Pro of. Work in the theory $\mathrm{M}+$ strong $\Pi_{1}$-collection. Let $\alpha$ be an ordinal. We shall use $\Sigma_{2}$-induction on $\omega$ to prove $(\forall n \in \omega) \exists f \Psi_{0}\left(n, f, \mathbf{V}_{\alpha}\right)$. It is clear that $\exists f \Psi_{0}\left(\varnothing, f, \mathbf{V}_{\alpha}\right)$ holds. Let $n \in \omega$ and suppose that $f$ is such that $\Psi_{0}\left(n, f, \mathbf{V}_{\alpha}\right)$ holds. Let $\beta$ be the ordinal such that $f(n)=\mathbf{V}_{\beta}$. Consider the $\Sigma_{1}$-formula $\psi(x, y)$ defined by

$$
\exists z \exists a \exists \ell\left((x=\langle a, \ell\rangle) \wedge(z=\langle y, a\rangle) \wedge\left(\ell=\ulcorner\varphi(u, v)\urcorner \text { where } \varphi \text { is } \Delta_{0}\right) \wedge \operatorname{Sat}_{\Delta_{0}}(\ell, z)\right) .
$$

Strong $\Sigma_{1}$-collection implies that there exists a $C$ such that

$$
\left(\forall x \in \mathbf{V}_{\beta} \times \omega\right)(\exists y \psi(x, y) \Rightarrow(\exists y \in C) \psi(x, y)) .
$$

Let $\gamma>\beta$ be such that $C \subseteq \mathbf{V}_{\gamma}$. Therefore, for all $\ell \in \omega$ and for all $a \in \mathbf{V}_{\beta}$,

$$
\text { if } \operatorname{Sat}_{\Sigma_{1}}(\ell, a) \text {, then }\left\langle\mathbf{V}_{\gamma}, \in\right\rangle \models \operatorname{Sat}_{\Sigma_{1}}(\ell, a) \text {. }
$$

It now follows that $g=f \cup\left\{\left\langle n+1, \mathbf{V}_{\gamma}\right\rangle\right\}$ satisfies $\Psi_{0}\left(n+1, g, \mathbf{V}_{\alpha}\right)$. The fact that $(\forall n \in \omega) \exists f \Psi_{0}\left(n, f, \mathbf{V}_{\alpha}\right)$ holds now follows by $\Sigma_{2}$-induction on $\omega$.

Lemma 4.4 The theory $M+$ strong $\Pi_{1}$-collection proves that for all ordinals $\alpha$, there exists a function $f$ with $\operatorname{dom}(f)=\omega$ such that for all $n \in \omega, f \uparrow(n+1)$ is an $n$-good $\langle 1, \alpha\rangle$-submodel approximation.

Pro of. Work in the theory $M+$ strong $\Pi_{1}$-collection. Using Lemma 4.3 and strong $\Sigma_{2}$-collection, we can find a set $B$ such that $(\forall n \in \omega)(\exists f \in B) \Psi_{0}\left(n, f, \mathbf{V}_{\alpha}\right)$ holds. Now, $\Sigma_{2}$-separation ensures that $D=\{f \in B \mid$ $\left.(\exists n \in \omega) \Psi_{0}\left(n, f, \mathbf{V}_{\alpha}\right)\right\}$ is a set. Let

$$
G=\{f \in D \mid(\forall k \in \operatorname{dom}(f))(\forall g \in D)(((k \in \operatorname{dom}(g)) \wedge(g(k) \neq f(k)) \Rightarrow f(k) \in g(k))\},
$$

which is a set. Now, for all $f_{1}, f_{2} \in G, f_{1}$ and $f_{2}$ agree on their common domain. Moreover, a straightforward internal induction using the fact that Lemma 4.3 holds shows that for all $n \in \omega,(\exists f \in G)(\operatorname{dom}(f)=n+1)$ holds. Therefore $g=\bigcup G$ is a function with domain $\omega$ such that for all $n \in \omega, \Psi_{0}\left(n, g \uparrow(n+1), \mathbf{V}_{\alpha}\right)$ holds.

We can now prove analogues of Lemmas $4.3 \& 4.4$ for the theories $\mathrm{M}+\Pi_{m}$-collection $+\Sigma_{m+1}$-induction on $\omega$ where $m \geq 2$.

Lemma 4.5 Let $m \geq 1$. The theory $\mathrm{M}+\Pi_{m+1}$-collection $+\Sigma_{m+2}$-induction on $\omega$ proves

(i) for all ordinals $\alpha$ and for all $n \in \omega$, there exists an $n$-good $\langle m+1, \alpha\rangle$-submodel approximation,

(ii) for all ordinals $\alpha$, there exists a function $f$ with $\operatorname{dom}(f)=\omega$ such that for all $n \in \omega, f\lceil(n+1)$ is an n-good $\langle m+1, \alpha\rangle$-submodel approximation. 
Pro of. We prove this lemma by external induction on $m$. We begin by proving the induction step. Suppose that (i) and (ii) of the lemma hold for $m=p \geq 1$. Work in the theory $\mathrm{M}+\Pi_{p+2}$-collection $+\Sigma_{p+3}$-induction on $\omega$. Let $\alpha$ be an ordinal. We shall use $\Sigma_{p+3}$-induction on $\omega$ to show that $(\forall n \in \omega) \exists f \Psi_{p+1}\left(n, f, \mathbf{V}_{\alpha}\right)$ holds. It is clear that $\exists f \Psi_{p+1}\left(\varnothing, f, \mathbf{V}_{\alpha}\right)$ holds. Let $n \in \omega$, and suppose that $\exists f \Psi_{p+1}\left(n, f, \mathbf{V}_{\alpha}\right)$ holds. Let $f$ be such that $\Psi_{p+1}\left(n, f, \mathbf{V}_{\alpha}\right)$. Let $\delta$ be the ordinal such that $f(n)=\mathbf{V}_{\delta}$. Consider the $\Sigma_{p+2}$-formula $\psi(x, y)$ defined by

$$
\exists z \exists a \exists \ell\left((x=\langle a, \ell\rangle) \wedge(z=\langle y, a\rangle) \wedge\left(\ell=\ulcorner\varphi(u, v)\urcorner \text { where } \varphi \text { is } \Pi_{p+1}\right) \wedge \operatorname{Sat}_{\Pi_{p+1}}(\ell, z)\right) .
$$

Strong $\Sigma_{p+2}$-collection implies that there exists a $C$ such that

$$
\left(\forall x \in \mathbf{V}_{\delta} \times \omega\right)(\exists y \psi(x, y) \Rightarrow(\exists y \in C) \psi(x, y)) .
$$

Let $\beta>\delta$ be such that $C \subseteq \mathbf{V}_{\beta}$. Now, using (ii) of the induction hypothesis, we can find a function $g$ with $\operatorname{dom}(g)=\omega$ such that for all $q \in \omega, \Psi_{p}\left(q, g \uparrow(q+1), \mathbf{V}_{\beta}\right)$. Now, let $\gamma>\beta$ be such that $\mathbf{V}_{\gamma}=\bigcup \operatorname{rng}(g)$. It follows from (iv) and (v) of Definition (4.1) that for all $\ell \in \omega$ and for all $a \in \mathbf{V}_{\gamma}$,

$$
\text { if }\left\langle\mathbf{V}_{\gamma}, \in\right\rangle \models \operatorname{Sat}_{\Pi_{p+1}}(\ell, a) \text {, then } \operatorname{Sat}_{\Pi_{p+1}}(\ell, a) \text {. }
$$

And, since $C \subseteq \mathbf{V}_{\beta} \subseteq \mathbf{V}_{\gamma}$, for all $\ell \in \omega$ and for all $a \in \mathbf{V}_{\delta}$,

$$
\text { if } \operatorname{Sat}_{\Sigma_{p+2}}(\ell, a) \text {, then }\left\langle\mathbf{V}_{\gamma}, \in\right\rangle \models \operatorname{Sat}_{\Sigma_{p+2}}(\ell, a) \text {. }
$$

Therefore, the function $h=f \cup\left\{\left\langle n+1, \mathbf{V}_{\gamma}\right\rangle\right\}$ satisfies $\Psi_{p+1}\left(n+1, h, \mathbf{V}_{\alpha}\right)$. The fact that $(\forall n \in \omega) \exists f \Psi_{p+1}\left(n, f, \mathbf{V}_{\alpha}\right)$ now follows from $\Sigma_{p+3}$-induction on $\omega$. This completes the induction step for (i). Turning our attention to (ii), we can use $\Pi_{p+2}$-collection to find a set $B$ such that $(\forall n \in \omega)(\exists f \in B) \Psi_{p+1}\left(n, f, \mathbf{V}_{\alpha}\right)$. Now, $\Pi_{p+2}$-separation ensures that $D=\left\{f \in B \mid(\exists n \in \omega) \Psi_{p+1}\left(n, f, \mathbf{V}_{\alpha}\right)\right\}$ is a set. Let

$$
G=\{f \in D \mid(\forall k \in \operatorname{dom}(f))(\forall g \in D)((k \in \operatorname{dom}(g)) \wedge(g(k) \neq f(k)) \Rightarrow f(k) \in g(k))\} .
$$

As in the proof of Lemma 4.4, if $f_{1}, f_{2} \in G$, then $f_{1}$ and $f_{2}$ agree on their common domain, and $(\forall n \in$ $\omega)(\exists f \in G)(\operatorname{dom}(f)=n+1)$. Therefore, $g=\bigcup G$ is a function with $\operatorname{dom}(g)=\omega$ such that for all $n \in \omega$, $\Psi_{p+1}\left(n, g \uparrow(n+1), \mathbf{V}_{\alpha}\right)$ holds. This completes the induction step for (ii). The base case of the induction on $m(m=1)$ follows from the same arguments used to prove the induction step with Lemma 4.4 replacing the induction hypothesis. This completes the proof of the lemma.

Using Lemma 4.5 we can show that for $m \geq 1, \mathrm{M}+\Pi_{m+1}$-collection $+\Sigma_{m+2}$-induction on $\omega$ proves that there exists a transitive model of $\mathrm{Z}+\Pi_{m}$-collection.

Theorem 4.6 Let $m \geq 1$. The theory $\mathrm{M}+\Pi_{m+1}$-collection $+\Sigma_{m+2}$-induction on $\omega$ proves that there exists a transitive models of $\mathrm{Z}+\Pi_{m}$-collection.

Proof. Work in the theory $\mathrm{M}+\Pi_{m+1}$-collection $+\Sigma_{m+2}$-induction on $\omega$. By Lemma 4.5(ii), there exists an $f$ such that $\operatorname{dom}(f)=\omega$, and for all $n \in \omega, f \uparrow(n+1)$ is an $n$-good $\langle m+1, \omega\rangle$-submodel approximation. Let $\beta$ be an ordinal such that $\mathbf{V}_{\beta}=\bigcup \operatorname{rng}(f)$. We claim that $\left\langle\mathbf{V}_{\beta}, \in\right\rangle$ is a set structure that satisfies $Z+\Pi_{m}$-collection. Since $\beta$ is a limit ordinal $>\omega$, it is immediate that $\left\langle\mathbf{V}_{\beta}, \in\right\rangle$ satisfies all of the axioms of Z. Let $\varphi(x, y, \vec{z})$ be a $\Pi_{m}$-formula. Let $\vec{a}, b \in \mathbf{V}_{\beta}$. Note that Definition 4.1 implies that $\mathbf{V}_{\beta}$ is a $\Pi_{m+1}$-elementary submodel of the universe, and for all $n \in \omega,\langle f(n), \in\rangle \prec_{m}\left\langle\mathbf{V}_{\beta}, \in\right\rangle$. Let $k \in \omega$ be such that $\vec{a}, b \in f(k)$. Now, it follows from Definition 4.1(v) that for all $x \in b$,

$$
\begin{array}{r}
\left\langle\mathbf{V}_{\beta}, \in\right\rangle \models \exists y \varphi(x, y, \vec{a}) \text { if and only if }\left\langle\mathbf{V}_{\beta}, \in\right\rangle \models(\exists y \in f(k+1)) \varphi^{\langle f(k+1), \in\rangle}(x, y, \vec{a}) \\
\text { if and only if }\left\langle\mathbf{V}_{\beta}, \in\right\rangle \models(\exists y \in f(k+1)) \varphi(x, y, \vec{a}) .
\end{array}
$$

Therefore

$$
\left\langle\mathbf{V}_{\beta}, \in\right\rangle \models(\forall x \in b)(\exists y \varphi(x, y, \vec{a}) \Rightarrow(\exists y \in f(k+1)) \varphi(x, y, \vec{a}))
$$

and so $\left\langle\mathbf{V}_{\beta}, \in\right\rangle$ satisfies strong $\Pi_{m}$-collection. Since $\left\langle\mathbf{V}_{\beta}, \in\right\rangle$ is a transitive set structure, we can conclude that $\mathrm{M}+\Pi_{m+1}$-collection $+\Sigma_{m+2}$-induction on $\omega$ proves that there exists a transitive model of $\mathrm{Z}+\Pi_{m}$-collection. 
We now turn to generalising Theorem 3.5 to show that for all $m \geq 1$, the theories $\mathrm{M}+$ strong $\Pi_{m}$-collection and $\mathrm{M}+\Pi_{m+1}$-collection have the same consistency strength. The key ingredient for this result will be the fact that if $m \geq 1$ and $\mathcal{M}$ is a model of $\mathrm{M}+$ strong $\Pi_{m}$-collection, then for every standard natural number $n$, there exists an $n$-good $\langle m+1, \omega\rangle$-submodel approximation in $\mathcal{M}$.

Lemma 4.7 Let $m \geq 1$ and let $\mathcal{M} \models \mathrm{M}+$ strong $\Pi_{m}$-collection. For all $n \in \omega$ and for all $\alpha \in \operatorname{Ord}^{\mathcal{M}}$, $\mathcal{M} \models \exists f(f$ is an $n$-good $\langle m+1, \alpha\rangle$-submodel approximation $)$.

Proof. Let $\alpha \in \operatorname{Ord}^{\mathcal{M}}$. We prove the lemma by external induction on $n$. It is clear that $\mathcal{M} \models \exists f(f$ is a 0 -good $\langle m+1, \alpha\rangle$-submodel approximation). Suppose that $p \in \omega$ and $f \in M$ are such that $\mathcal{M}=(f$ is a $p$-good $\langle m+1, \alpha\rangle$-submodel approximation). Work inside $\mathcal{M}$. Let $\mathbf{V}_{\delta}$ be the rank such that $f(p)=\mathbf{V}_{\delta}$. Consider the $\Pi_{m}$-formula $\psi(x, y)$ defined by

$$
(x=\langle a, \ell\rangle) \wedge\left(\ell=\ulcorner\varphi(u, v)\urcorner \text { where } \varphi \text { is } \Pi_{m}\right) \wedge \operatorname{Sat}_{\Pi_{m}}(\ell,\langle y, a\rangle) .
$$

Strong $\Pi_{m}$-collection implies that there is a set $C$ such that

$$
\left(\forall x \in \mathbf{V}_{\delta} \times \omega\right)(\exists y \psi(x, y) \Rightarrow(\exists y \in C) \psi(x, y)) .
$$

Let $\gamma>\delta$ be such that $C \subseteq \mathbf{V}_{\gamma}$. Using Lemma 4.4 (if $m=1$ ) or Lemma 4.5 (if $m>1$ ), we can find a function $g$ with $\operatorname{dom}(g)=\omega$ such that for all $k \in \omega, g \uparrow(k+1)$ is a $k$-good $\langle m, \gamma\rangle$-submodel approximation. Let $\beta$ be such that $\mathbf{V}_{\beta}=\bigcup \operatorname{rng}(g)$. It follows that for all $\ell \in \omega$ and for all $a \in \mathbf{V}_{\beta}$, if $\left\langle\mathbf{V}_{\beta}, \in\right\rangle \models \operatorname{Sat}_{\Pi_{m}}(\ell, a)$, then $\operatorname{Sat}_{\Pi_{m}}(\ell, a)$. And, since $C \subseteq \mathbf{V}_{\beta}$, for all $\ell \in \omega$ and for all $a \in \mathbf{V}_{\delta}$, if $\operatorname{Sat}_{\Sigma_{m+1}}(\ell, a)$, then $\left\langle\mathbf{V}_{\beta}, \in\right\rangle \models \operatorname{Sat}_{\Sigma_{m+1}}(\ell, a)$. Therefore, $h=f \cup\left\{\left\langle p+1, \mathbf{V}_{\beta}\right\rangle\right\}$ is a $p+1$-good $\langle m+1, \alpha\rangle$-submodel approximation. This concludes the proof of the induction step and the lemma.

We now use a generalisation of the construction used is the proof of Theorem 3.5 to obtain a model $\mathrm{M}+$ $\Pi_{m+1}$-collection from a model of $\mathrm{M}+$ strong $\Pi_{m}$-collection.

Theorem 4.8 Let $m \geq 1$.

(i) The theory $\mathrm{M}+\Pi_{m+1}$-collection is $\Pi_{m+3}$-conservative over the theory $\mathrm{M}+$ strong $\Pi_{m}$-collection.

(ii) The theory $\mathrm{Mac}+\Pi_{m+1}$-collection is $\Pi_{m+3}$-conservative over the theory Mac + strong $\Pi_{m}$-collection.

Pro of. To prove (i) it is sufficient to show that every $\Sigma_{m+3}$-sentence that is consistent with $M+$ strong $\Pi_{m}$-collection is also consistent with $M+\Pi_{m+1}$-collection. Suppose that $\exists \vec{x} \forall \vec{y} \vartheta(\vec{x}, \vec{y})$, where $\vartheta(\vec{x}, \vec{y})$ is a $\Sigma_{m+1}$-formulae, is consistent with $\mathrm{M}+$ strong $\Pi_{m}$-collection. Let $\mathcal{M}=\langle M, \in \mathcal{M}\rangle$ be a recursively saturated model of $\mathrm{M}+$ strong $\Pi_{m}$-collection $+\exists \vec{x} \forall \vec{y} \vartheta(\vec{x}, \vec{y})$. Let $\vec{a} \in M$ be such $\mathcal{M} \models \forall \vec{y} \vartheta(\vec{a}, \vec{y})$ and let $\alpha \in M$ be an ordinal such that $\vec{a} \in\left(\mathbf{V}_{\alpha}^{\mathcal{M}}\right)^{*}$. Consider the type

$$
\Xi(x, u)=\{x \in \omega\} \cup\{x>n \mid n \in \omega\} \cup\{\exists f(f \text { is an } x \text {-good }\langle m+1, \alpha\rangle \text {-submodel approximation })\} .
$$

By Lemma 4.7, $\Xi(x, u)$ is finitely realised in $\mathcal{M}$, and so there exists $k \in M$ such that $\Xi(k, u)$ is satisfied in $\mathcal{M}$. Note that $k$ is a nonstandard element of $\omega^{\mathcal{M}}$. Let $f \in M$ be such that $\mathcal{M} \models(f$ is a $k$-good $\langle m+1, \alpha\rangle$-submodel approximation). Define $\mathcal{N}=\left\langle N, \in^{\mathcal{N}}\right\rangle$ by

$$
N=\bigcup_{n \in \omega} f\left(n^{\mathcal{M}}\right)^{*} \text { and } \in^{\mathcal{N}} \text { is the restriction of } \in^{\mathcal{M}} \text { to } N .
$$

We claim that $\mathcal{N}$ satisfies $\mathrm{M}+\Pi_{m+1}$-collection $+\exists \vec{x} \forall \vec{y} \vartheta(\vec{x}, \vec{y})$. Note that $\mathcal{N} \subseteq_{\mathrm{e}}^{\wp} \mathcal{M}$. It follows from the fact that $f$ is an $k$-good $\langle m+1, \alpha\rangle$-submodel approximation that $\mathcal{N} \models \mathrm{M}$ and for all $x \in N, \wp^{\mathcal{N}}(x)=\wp^{\mathcal{M}}(x)$. Moreover, Definition 4.1(iv) implies that $\mathcal{N} \prec_{m+1} \mathcal{M}$. Therefore, since $\vec{a} \in N, \mathcal{N} \models \exists \vec{x} \forall \vec{y} \vartheta(\vec{x}, \vec{y})$.

We are left to show that $\Pi_{m+1}$-collection holds in $\mathcal{N}$. Using exactly the same reasoning that was used in the proof of Theorem 3.5, we can see that if $C \in M$ is such that $C^{*} \subseteq N$, then $C \in N$. Now, let $\varphi(x, y, \vec{z})$ be a $\Pi_{m+1}$-formula. Let $\vec{d}, b \in N$ be such that $\mathcal{N}=(\forall x \in b) \exists y \varphi(x, y, \vec{d})$ The following formula is a Bool( $\left(\Pi_{m+1}\right)$ formula with parameters $\vec{d}, k$ and $f$ :

$$
\varphi(x, y, \vec{d}) \wedge(\forall n \in k)(y \notin f(n) \Rightarrow \neg(\exists w \in f(n)) \varphi(x, w, \vec{d})) .
$$


And, since $\mathcal{N} \prec_{m+1} \mathcal{M}$,

$$
\mathcal{M} \models(\forall x \in b)(\exists y \in f(k))(\varphi(x, y, \vec{d}) \wedge(\forall n \in k)(y \notin f(n) \Rightarrow \neg(\exists w \in f(n)) \varphi(x, w, \vec{d}))) .
$$

Working inside $\mathcal{M}, \operatorname{Bool}\left(\Pi_{m+1}\right)$-separation (Lemma 2.3) implies that

$$
C=\{\langle x, y\rangle \in b \times f(k) \mid \varphi(x, y, \vec{d}) \wedge(\forall n \in k)(y \notin f(n) \Rightarrow \neg(\exists w \in f(n)) \varphi(x, w, \vec{d}))
$$

is a set. And, the fact that $\mathcal{N} \prec_{m+1} \mathcal{M}$ ensures that $C^{*} \subseteq N$. Therefore $C \in N$. Working inside $\mathcal{N}$, let $B=\operatorname{rng}(C)$. So, $\mathcal{N}=(\forall x \in b)(\exists y \in B) \varphi(x, y, \vec{d})$, which shows that $\mathcal{N}=\Pi_{m+1}$-collection.

To see that (ii) holds observe that if the Axiom of Choice holds in $\mathcal{M}$ in the proof of (i), then it also holds in $\mathcal{N}$.

Corollary 4.9 If $\mathrm{M}+$ strong $\Pi_{m}$-collection is consistent, then so is $\mathrm{M}+\Pi_{m+1}$-collection.

Theorem 4.6 and Corollary 4.9 yield:

Corollary 4.10 If $m \geq 1$, then $\mathrm{M}+\Pi_{m+1}$-collection $\vdash \operatorname{Con}\left(\mathrm{M}+\Pi_{m}\right.$-collection).

These results also reveal the limitations of the theory $\mathrm{M}+\Pi_{m}$-collection when $m \geq 2$.

Corollary 4.11 If $m \geq 1$, then $\mathrm{M}+\Pi_{m+1}$-collection $\nvdash \Sigma_{m+2}$-induction on $\omega$.

Proof. One can easily verify that by starting with a model of $M+$ strong $\Pi_{m}$-collection $+\neg \operatorname{Con}(Z+$ $\Pi_{m}$-collection) in the proof of Theorem 4.8, one obtains a model of $M+\Pi_{m+1}$-collection $+\neg \operatorname{Con}(Z+$ $\Pi_{m}$-collection). If $\mathrm{M}+\Pi_{m+1}$-collection proves $\Sigma_{m+2}$-induction, then, by Theorem 4.6, this model would also satisfy $\operatorname{Con}\left(Z+\Pi_{m}\right.$-collection), which is a contradiction.

The proof of [7, Proposition 9.20] shows that there is an instance of $\Sigma_{2}$-induction on $\omega$ that coupled with the theory $\mathrm{M}$ proves the consistency of Mac. Therefore, by observing that the proof of Theorem 3.5 can be used to obtain a model of MOST $+\Pi_{1}$-collection $+\neg$ Con(MOST), we can see that there is an instance of $\Sigma_{2}$-induction on $\omega$ that is not provable in MOST $+\Pi_{1}$-collection. Therefore Corollary 4.11 also holds when $m=0$.

\section{The strength of $\Pi_{n}$-collection over $\mathrm{KPI}+\mathrm{V}=\mathrm{L}$}

In this section we show that the techniques developed in $\S \S 3 \& 4$ can be adapted to reveal the relative strengths of fragments of the collection scheme over the base theory $\mathrm{KPI}+\mathbf{V}=\mathbf{L}$. This is achieved by replacing the levels of the $V$-hierarchy in Definition 4.1 by levels of the L-hierarchy. if

Definition 5.1 Let $n, m \in \omega$, and let $\alpha$ be an ordinal. We say that $f$ is an $n$-good $\langle m+1, \alpha\rangle$-L-approximation

(i) $f$ is a function and $\operatorname{dom}(f)=n+1$,

(ii) $f(\varnothing)=\mathbf{L}_{\alpha}$,

(iii) $(\forall k \in n+1) \exists \beta\left((\beta\right.$ is an ordinal $\left.) \wedge f(k)=\mathbf{L}_{\beta}\right)$,

(iv) $(\forall k \in n)(\forall \ell \in \omega)(\forall a \in f(k+1))\left(\left(\langle f(k+1), \in\rangle \models \operatorname{Sat}_{\Pi_{m}}(\ell, a)\right) \Rightarrow \operatorname{Sat}_{\Pi_{m}}(\ell, a)\right)$, and

(v) $(\forall k \in n)(\forall \ell \in \omega)(\forall a \in f(k))\left(\operatorname{Sat}_{\Sigma_{m+1}}(\ell, a) \Rightarrow\left(\langle f(k+1), \in\rangle \models \operatorname{Sat}_{\Sigma_{m+1}}(\ell, a)\right)\right)$.

Note that the only difference between Definitions $4.1 \& 5.1$ are that the references to levels of the $V$-hierarchy in clauses (ii) and (iii) of Definition 4.1 have been replaced by level of the L-hierarchy in Definition 5.1. It should be clear that the expression " $f(\varnothing)=\mathbf{L}_{\alpha}$ " remains $\Delta_{0}$ with parameters $f$ and $\mathbf{L}_{\alpha}$, and, in light of Theorem 2.11, the expression " $(\forall k \in n+1) \exists \beta\left((\beta\right.$ is an ordinal $\left.) \wedge f(k)=\mathbf{L}_{\beta}\right)$ " is equivalent to a $\Sigma_{1}$-formula with parameters $f$ and $n$ in the theory KPI. As we did in $\S 4$, we introduce specific notion for formulae that express that $f$ is an $n$-good $\langle m+1, \alpha\rangle$-L-approximation. 
Definition 5.2 Let $\alpha$ be an ordinal and let $m \in \omega$. We write $\Psi_{m}^{*}\left(n, f, \mathbf{L}_{\alpha}\right)$ for the formula, with free variables $f$ and $n$, and parameter $\mathbf{L}_{\alpha}$, that the theory $\mathrm{KPI}$ proves asserts that $f$ in an $n$-good $\langle m+1, \alpha\rangle$-L-approximation, and such that $\Psi_{0}^{*}\left(n, f, \mathbf{L}_{\alpha}\right)$ is $\operatorname{Bool}\left(\Sigma_{2}\right)$, and if $m>0, \Psi_{m}^{*}\left(n, f, \mathbf{L}_{\alpha}\right)$ is $\Pi_{m+1}$.

Using the same arguments as we used in the proofs of Lemmas $4.3 \& 4.4$ we obtain:

Lemma 5.3 The theory $\mathrm{KPI}+\mathbf{V}=\mathbf{L}+\Pi_{1}$-collection $+\Sigma_{2}$-induction on $\omega$ proves that for all ordinals $\alpha$ and for all $n \in \omega$, there exists an $n$-good $\langle 1, \alpha\rangle$-L-approximation.

Lemma 5.4 The theory $\mathrm{KPI}+\mathbf{V}=\mathbf{L}+\Pi_{1}$-collection $+\Sigma_{2}$-induction on $\omega$ proves that for all ordinals $\alpha$, there exists a function $f$ with $\operatorname{dom}(f)=\omega$ such that for all $n \in \omega, f \uparrow(n+1)$ in an n-good $\langle 1, \alpha\rangle$-L-approximation.

Lemmas $5.3 \& 5.4$ now provide the base case of an induction argument that proves an analogue of Lemma 4.5.

Lemma 5.5 Let $m \in \omega$. The theory $\mathrm{KPI}+\mathbf{V}=\mathbf{L}+\Pi_{m+1}$-collection $+\Sigma_{m+2}$-induction proves

(i) for all ordinals $\alpha$ and for all $n \in \omega$, there exists an $n$-good $\langle m+1, \alpha\rangle$-L-approximation,

(ii) for all ordinals $\alpha$, there exists a function $f$ with $\operatorname{dom}(f)=\omega$ such that for all $n \in \omega, f \uparrow(n+1)$ is an n-good $\langle m+1, \alpha\rangle$-L-approximation.

Lemma 5.5 provides the key ingredient for showing that the theory $\mathrm{KPI}+\mathbf{V}=\mathbf{L}+\Pi_{m+1}$-collection $+\Sigma_{m+2}$-induction on $\omega$ proves the consistency of the theory KPI $+\mathbf{V}=\mathbf{L}+$ strong $\Pi_{m}$-collection $+\Pi_{\infty}$-foundation.

Theorem 5.6 Let $m \in \omega$. The theory $\mathrm{KPI}+\mathbf{V}=\mathbf{L}+\Pi_{m+1}$-collection $+\Sigma_{m+2}$-induction on $\omega$ proves that there exists a transitive model of $\mathrm{KPI}+\mathbf{V}=\mathbf{L}+$ strong $\Pi_{m}$-collection $+\Pi_{\infty}$-foundation.

Proof. Work in the theory KPI $+\mathbf{V}=\mathbf{L}+\Pi_{m+1}$-collection $+\Sigma_{m+2}$-induction on $\omega$. By Lemma 5.5(ii), there exists $f$ such that $\operatorname{dom}(f)=\omega$, and for all $n \in \omega, f \uparrow(n+1)$ is an $n$-good $\langle m+1, \omega\rangle$-L-approximation. Let $\beta$ be an ordinal such that $\mathbf{L}_{\beta}=\bigcup \operatorname{rng}(f)$. We claim that $\left\langle\mathbf{L}_{\beta}, \in\right\rangle$ is a set structure that satisfies $\mathrm{KPI}+$ strong $\Pi_{m}$-collection $+\Pi_{\infty}$-foundation (= $\mathrm{M}^{-}+$strong $\Pi_{m}$-collection $+\Pi_{\infty}$-foundation). Note that, since $\beta$ is a limit ordinal, $\mathbf{L}_{\beta}$ is a transitive set that is closed under Gödel operations. Therefore $\left\langle\mathbf{L}_{\beta}, \in\right\rangle$ satisfies all of the axioms of $\mathrm{M}^{-}$. Let $\varphi(x, \vec{z})$ be a $\Pi_{\infty}$-formula and let $\vec{a} \in \mathbf{L}_{\beta}$. Separation in the theory KPI implies that $A=\left\{x \in \mathbf{L}_{\beta} \mid\left\langle\mathbf{L}_{\beta}, \in\right\rangle \models \varphi(x, \vec{a})\right\}$ is a set. Therefore, set foundation in KPI, implies that if $A \neq \varnothing$, then $A$ has an $\in$-least element. This shows that $\left\langle\mathbf{L}_{\beta}, \in\right\rangle$ satisfies $\Pi_{\infty}$-foundation. Finally, identical reasoning to that used in the proof of Theorem 4.6 shows that $\left\langle\mathbf{L}_{\beta}, \in\right\rangle$ satisfies strong $\Pi_{m}$-collection. Since $\left\langle\mathbf{L}_{\beta}, \in\right\rangle$ is a transitive set structure, we can conclude that $\mathrm{KPI}+\Pi_{m+1}$-collection $+\Sigma_{m+2}$-induction on $\omega$ proves that there exists a transitive models of $\mathrm{KPI}+$ strong $\Pi_{m}$-collection $+\Pi_{\infty}$-foundation $+\mathbf{V}=\mathbf{L}$.

We next turn indicating how the proof of Theorem 4.8 can be adapted to obtain an analogue of this result with the base theory M replaced by KPI $+\mathbf{V}=\mathbf{L}$. The same argument used in the proof of Lemma 4.7 can be used to prove the following:

Lemma 5.7 Let $m \in \omega$ and let $\mathcal{M} \models \mathrm{KPI}+\mathbf{V}=\mathbf{L}+$ strong $\Pi_{m}$-collection. For all $n \in \omega$ and for all $\alpha \in \operatorname{Ord}^{\mathcal{M}}$, we have $\mathcal{M}=\exists f(f$ is an $n$-good $\langle m+1, \alpha\rangle$-L-approximation $)$.

Lemma 5.7 yields an analogue of Theorem 4.8.

Theorem 5.8 Let $m \in \omega$.

(i) The theory $\mathrm{KPI}+\mathbf{V}=\mathbf{L}+\Pi_{m+1}$-collection is $\Pi_{m+3}$-conservative over the theory $\mathrm{KPI}+\mathbf{V}=\mathbf{L}+$ strong $\Pi_{m}$-collection.

(ii) If $\mathrm{KPI}+\mathbf{V}=\mathbf{L}+$ strong $\Pi_{m}$-collection is consistent, then so is $\mathrm{KPI}+\mathbf{V}=\mathbf{L}+\Pi_{m+1}$-collection.

Theorems $5.6 \& 5.8$ yield:

Corollary 5.9 If $m \geq 1$, then $\mathrm{KPI}+\mathbf{V}=\mathbf{L}+\Pi_{m+1}$-collection $\vdash \mathrm{Con}\left(\mathrm{KPI}+\mathbf{V}=\mathbf{L}+\Pi_{m}\right.$-collection).

Question 5.10 Does the theory $\mathrm{KPI}+\mathbf{V}=\mathbf{L}+$ strong $\Pi_{0}$-collection prove the consistency of $\mathrm{KPI}$ ?

I am grateful to Ali Enayat for the following observation: The proofs of Theorems 3.5, 3.10, 4.8 and 5.8 can all be formalised in the subsystem of second order arithmetic $\mathrm{WKL}_{0}$. The fact that $\mathrm{WKL}_{0}$ is conservative over Primitive Recursive Arithmetic (PRA) for sentences that are $\Pi_{2}$ sentences of arithmetic (cf. [9, Theorem IX.3.16]), then shows that all of these results are theorems of PRA. 
Acknowledgements I am very grateful to Adrian Mathias and Ali Enayat for their helpful comments on earlier drafts of this paper. In particular, Ali Enayat's observations led to the strengthening of Theorems 3.5, 3.10, 4.8 and 5.8. I would also like to thank the anonymous referee for their careful reading of this paper and their thoughtful suggestions.

\section{References}

[1] J. Barwise, Admissible Sets and Structures, an Approach to Definability Theory, Perspectives in Mathematical Logic (Springer, 1975).

[2] T. Forster and R. Kaye, End-extensions preserving power set, J. Symb. Log. 56(1), 323-328 (1991).

[3] H. M. Friedman, Countable models of set theories, in: Cambridge Summer School in Mathematical Logic, held in Cambridge, U. K., August 1-21, 1971, edited by A. R. D. Mathias and H. Rogers Jr., Springer Lecture Notes in Mathematics Vol. 337 (Springer, 1973), pp. 539-573.

[4] S.-D. Friedman, W. Li, and T. L. Wong, Fragments of Kripke-Platek set theory and the metamathematics of $\alpha$-recursion theory, Arch. Math. Log. 55(7), 899-924 (2016).

[5] Z. McKenzie, Automorphisms of models of set theory and extensions of NFU, Ann. Pure Appl. Log. 166, 601-638 (2015).

[6] A. R. D. Mathias, Notes on Set Theory, unpublished notes on lectures on admissibility and constructibility at the University of Wisconsin, Madison, 1969.

[7] A. R. D. Mathias, The strength of Mac Lane set theory, Ann. Pure Appl. Log. 110, 107-234 (2001).

[8] J.-P. Ressayre, Modèles non standard et sous-systèmes remarquables de ZF, in: J.-P. Ressayre and A. Wilkie, Modèles Non Standard en Arithmétique et Théorie des Ensembles, Publications Mathématiques de l’Université Paris VII Vol. 22 (U.E.R. de Mathématiques, 1987), pp. 47-147.

[9] S. G. Simpson, Subsystems of Second Order Arithmetic, Perspectives in Logic, second ed. (Cambridge University Press, 2009).

[10] M. Takahashi, $\tilde{\Delta}_{1}$-definability in set theory, in: Conference in Mathematical Logic_London ' 70 , Proceedings of a Conference, Bedford College, London, 24-28 August 1970, edited by W. Hodges, Lecture Notes in Mathematics Vol. 255 (Springer, 1972), pp. 281-304. 